\title{
Integrated insulin pump therapy with continuous glucose monitoring for improved adherence: technology update
}

This article was published in the following Dove Press journal:

Patient Preference and Adherence

7 September 2015

Number of times this article has been viewed

\section{Andrea Tumminia' \\ Laura Sciacca' \\ Lucia Frittitta' \\ Sebastiano Squatrito' \\ Riccardo Vigneri ${ }^{2}$ \\ Rosario Le Moli' \\ Letizia Tomaselli²}

'Endocrinology, Department of Clinical and Experimental Medicine, University of Catania, GaribaldiNesima Hospital, Catania, Italy; ${ }^{2}$ Endocrinology, Garibaldi-Nesima Hospital, Catania, Italy
Correspondence: Andrea Tumminia Endocrinology, Department of Clinical and Experimental Medicine, University of Catania, Garibaldi-Nesima Hospital, Via Palermo 636, 95I22, Catania, Italy Tel +393384755108

Fax +39095472988

Email andreatumminia@libero.it
Abstract: Insulin pump therapy combined with real-time continuous glucose monitoring, known as sensor-augmented pump (SAP) therapy, has been shown to improve metabolic control and to reduce the rate of hypoglycemia in adults with type 1 diabetes compared to multiple daily injections or standard continuous subcutaneous insulin infusion. Glycemic variability is also reduced in patients on SAP therapy. This approach allows patients to monitor their glucose levels being informed of glycemic concentration and trend. Trained diabetic patients, therefore, can appropriately modify insulin infusion and/or carbohydrate intake in order to prevent hypo- or hyperglycemia. For these reasons, SAP therapy is now considered the gold standard for type 1 diabetes treatment. To be clinically effective, however, devices and techniques using advanced technology should not only have the potential to theoretically ameliorate metabolic control, but also be well accepted by patients in terms of satisfaction and health-related quality of life, because these factors will improve treatment adherence and consequently overall outcome. SAP therapy is generally well tolerated by patients; however, many clinical trials have identified significant noncompliance in the use of this device, most notably in the pediatric and adolescent populations. In this review we aim to analyze the main reasons for good or poor adherence to SAP therapy and to provide useful tips in order to fully benefit from this kind of novel therapeutic approach.

Keywords: sensor-augmented insulin pump therapy, type 1 diabetes, quality of life, patient adherence, continuous subcutaneous insulin infusion

\section{General considerations on real-time continuous glucose monitoring (RT-CGM) and sensor- augmented pump (SAP) therapy}

Real-time continuous glucose monitoring (RT-CGM) systems represent an important advancement in diabetes technology that can facilitate optimal glucose control in type 1 diabetic patients (T1D). Several randomized and controlled clinical trials have demonstrated the safety and efficacy of RT-CGM in both suboptimally and wellcontrolled T1D patients. ${ }^{1-6}$ In addition, further studies demonstrated the superiority of SAP therapy, which integrates an insulin pump with the RT-CGM system (Figure 1 ), versus multiple daily injections, with a mean decrease of $0.8 \%-1.2 \%$ in glycated hemoglobin $\left(\mathrm{HbA}_{1 \mathrm{c}}\right)$ levels, without increase of hypoglycemia. ${ }^{7,8}$ In all these trials, RT-CGM benefits were correlated with the time spent wearing the sensor and with more advanced age. ${ }^{9}$ Actually, a sensor use of at least $60 \%-70 \%$ of the total time is required to reach the clinical benefits of sensor-augmented pump (SAP) therapy., ${ }^{2,9}$

In daily practice, SAP therapy is indicated for patients who cannot achieve good metabolic control on continuous subcutaneous insulin infusion (CSII) alone, who 

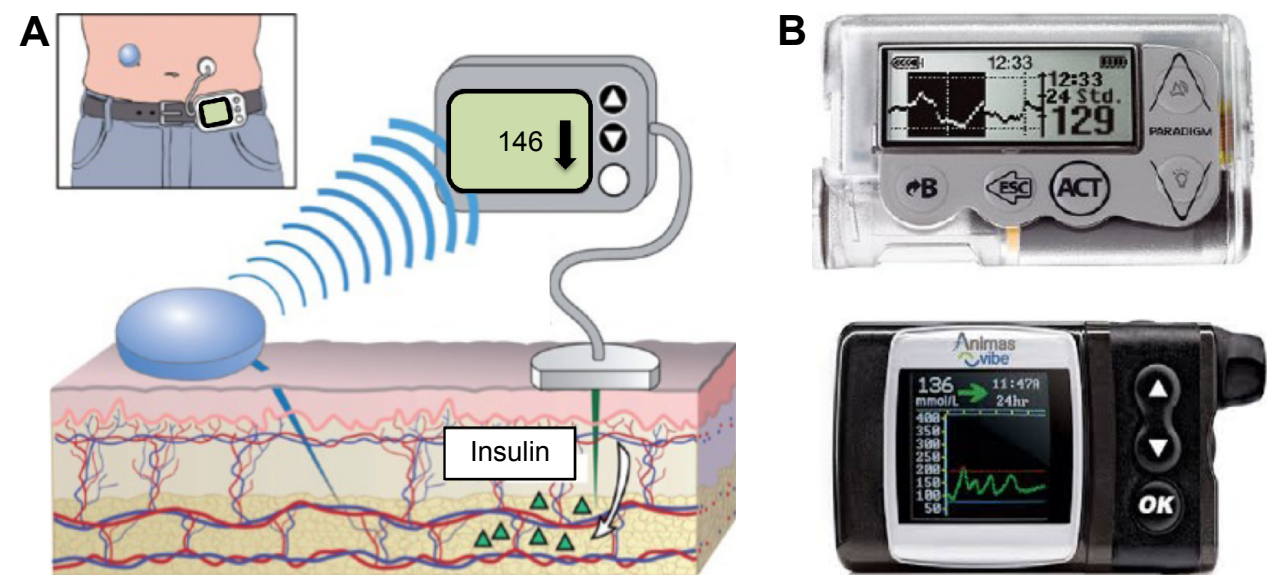

Figure I Sensor-augmented pump therapy.

Notes: (A) Real-time glucose sensor, sitting on the skin surface with its tip inserted in the tissue interstitial fluid, measures glucose levels. The glucose value is transmitted to the insulin pump by radio frequency. (B) Current available devices for sensor-augmented insulin pump therapy: Medtronic Paradigm VEO (Northridge, CA, USA) (top); Animas VIBE (West Chester, PA, USA) (bottom).

have a history of severe hypoglycemia and/or hypoglycemia unawareness, or who require increased flexibility in their everyday lives while maintaining or even improving their $\mathrm{HbA}_{1 \mathrm{c}}$ levels. ${ }^{10-12}$

To be really effective, SAP therapy should not only be able to cause $\mathrm{HbA}_{1 \mathrm{c}}$ reduction, improve glucose variability and, decrease hypoglycemic episodes, but should also consider patient-dependent outcomes such as treatment satisfaction, family relationships, lifestyle flexibility, anxiety, fear of diabetes complications, and treatment expectations. ${ }^{13-16}$ Healthrelated quality of life (QoL) is a critical outcome because treatment satisfaction directly affects patient acceptance. Previous studies, reporting that RT-CGM systems improve clinical outcomes only when they are used consistently, ${ }^{2,7,9}$ confirm the critical role of patient acceptance for optimizing the benefits of all new treatments or technologies. Few works have systematically examined the impact of SAP therapy on patient-related outcomes. However, surveys from patients on SAP therapy showed that the most common perceived benefits are prevention of hypoglycemia and decrease in hypoglycemia-related anxiety. ${ }^{13,15}$

\section{Factors influencing the efficacy of SAP therapy}

Many practical factors can strongly influence the efficacy of SAP therapy. We must specify that, even if technology could deeply affect the patients' lifestyle, it would be useless if not supported by a robust and continuous educational approach as demonstrated by the major randomized controlled trials on SAP therapy. ${ }^{2,6,7}$

Perhaps the most important characteristic for predicting the best results is the patient motivation and the will and capacity to change certain behaviors regarding diabetes management. We will discuss, therefore, the main factors involved in the success (or failure) of SAP therapy (Table 1).

Table I Factors involved in the success (or failure) of SAP therapy

Patient-related
- Motivation to change previous behaviors regarding diabetes
management.
- Frequency of sensor use (at least $60 \%-70 \%$ of the total time).
- Right interpretation of trends on RT-CGM receiver (glucose
trend arrows).
- Awareness of the lag-time period (RT-CGM devices measuring
interstitial fluid glucose concentration, exhibit $3-12$ minutes
time delay when compared to capillary blood glucose).

Health care giver-related

- Proper patient selection.

- Training on diabetes management (eg, self-management of blood glucose monitoring, dietary education, carbohydrate counting, etc).

- Training on technical devices (glucometer, insulin pump, RT-CGM system).

- Instruction to patients regarding the use of specific algorithms to adjust their insulin regimen based on glucose trends.

- Involvement of parents in the educational program (children and adolescents).

Abbreviations: RT-CGM, real-time continuous glucose monitoring; SAP, sensor-augmented insulin pump. 


\section{Patient selection, motivation, and training}

Patient selection is, in our opinion, the first and most important step for prescribing SAP therapy. It is essential to choose patients who are motivated to improve their knowledge about the disease and willing to actively collaborate with health care givers in the educational program (ie, self-management of blood glucose monitoring, dietary education, carbohydrate counting). Patients under SAP therapy are required to have a good understanding of the basic principles of insulin therapy for T1D. ${ }^{17,18}$ Well-trained patients will most likely seek continuous protection against hypo- or hyperglycemia, while patients who do not understand or practice the basics of intensive insulin treatment are unlikely to obtain significant advantages by the adoption of SAP therapy. Therefore, appropriate patient selection is a critical factor to predict SAP therapy success.

Moreover, SAP therapy success is dependent on health care providers' capacity to ensure that selected patients are appropriately trained not only on diabetes management but also on the technical devices' functioning (glucometer, CGM receiver, insulin pump) in order to understand how to correctly use them for maximum benefit.

\section{Frequency of sensor use}

The second major factor for predicting SAP therapy success is the frequency of sensor use, closely related to the previous criterion. Wearing the device at least $60 \%-70 \%$ of the total time predicted significant $\mathrm{HbA}_{1 \mathrm{c}}$ reduction in the STAR 1 study.$^{19}$ Furthermore, in the JDRF randomized controlled trial, the group with the greatest success (those over 25 years old) also had the greatest frequency of sensor use: $83 \%$ of the patients wore the device at least 6 days each week. ${ }^{2}$ Conversely, worst outcome was observed in patients aged 15-24 years, where only $30 \%$ of the subjects wore the device properly. In both of the trials, post hoc analyses concluded that, regardless of age, subjects who were not committed to wearing the device had little benefit, ${ }^{20}$ whereas those who consistently used the device had significant improvements in $\mathrm{HbA}_{1 \mathrm{c}}$. Also, the STAR 3 study, the most important randomized controlled trial on SAP therapy, demonstrated that the increased frequency of sensor use was strongly associated with a greater reduction of the $\mathrm{HbA}_{1 \mathrm{c}}$ level at 1 year. $^{7}$

The conclusion is that SAP therapy works only when it is used appropriately and consistently, as with most technologies.

\section{Glucose trends and lag time}

Once appropriate patients have been selected and trained, the major challenge is to get the maximum beneficial effect from
SAP therapy with regard to the right interpretation of glucose trends, displayed as arrows on the CGM receiver (Figure 2). Patient instruction in the use of evidence-based algorithms for adjusting insulin regimens on the basis of glucose trends can be extremely helpful. One way is to use the rate of glucose change to alter the premeal bolus, with a $1-2 \mathrm{mg} / \mathrm{dL} / \mathrm{min}$ change indicating the need for a $10 \%$ adjustment in bolus dosing (a single arrow up or down), while adjusting the bolus dose by $20 \%$ when the CGM system alerts that glucose levels are more rapidly changing (more than $2 \mathrm{mg} / \mathrm{dL} / \mathrm{min}$, a double arrow up or down, Figure 2). ${ }^{21}$

Another important factor is patient awareness of the existing lag time between blood glucose and interstitial fluid glucose. In fact, due to the use of the enzymatic sensor inserted subcutaneously, the RT-CGM device estimates blood glucose by measuring the glucose concentration in the interstitial fluid. Signals acquired from the interstitial space are subsequently calibrated with capillary blood glucose samples. RT-CGM devices, therefore, indicate glucose levels with a time delay compared to capillary blood glucose. The delay (3-12 minutes) is due to the time required for glucose diffusion across the capillary endothelial barrier. ${ }^{22,23}$ Each patient initiating SAP therapy should be aware of these dynamics because they directly influence the interpretation of RT-CGM. On the basis of our clinical experience, we found that some patients may find it difficult to pay more attention to the glucose trend rather than to the specific value reported on the CGM receiver. Particularly during rapid glycemic changes, the actual blood glucose value may be significantly different from what is reported on the CGM receiver. If only reacting to this number, instead of measuring a capillary blood glucose value, the patient might give an inappropriate insulin dose or improperly introduce carbohydrates.
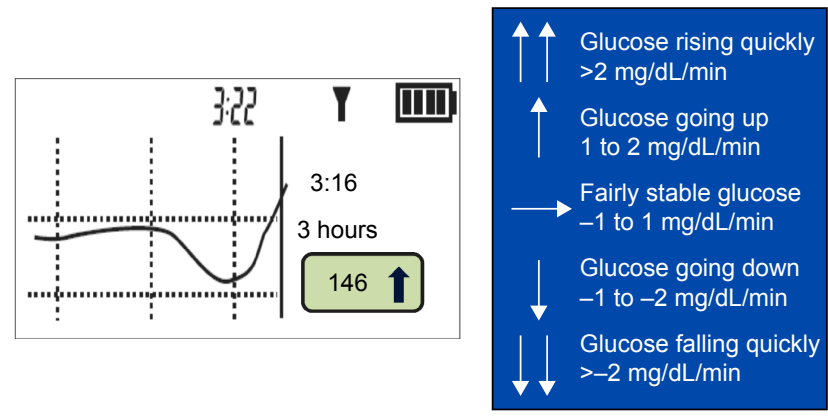

Figure 2 Glucose trend arrows.

Notes: Real-time continuous glucose monitoring data include "trend arrows" which indicate when the blood glucose is rapidly falling or rising, thus enabling the pump user to make immediate adjustments in insulin delivery to prevent subsequent hypoor hyperglycemia. 


\section{QoL and SAP therapy adherence in children and adolescents (the real challenge)}

Several studies tried to investigate the impact of SAP therapy on QoL. ${ }^{24-27}$ These trials showed that patient-reported QoL could be associated with the frequency of sensor use and the quality of achieved glycemic control. In the JDRF study, CGM satisfaction was higher for patients using RT-CGM 6 or more days per week. ${ }^{24}$ Patients' reporting satisfaction on SAP therapy has been particularly evaluated in a pediatric population, measuring QoL in both children and their parents. The great interest in pediatric patients is due to the fact that children and adolescents often show a very low compliance to this kind of treatment. In the DirecNet study, only $41 \%$ of children used CGM at least 6 or more days per week at the end of study (26 weeks). ${ }^{25}$ In terms of QoL and treatment satisfaction, higher parents' scores on a self-management survey ${ }^{24}$ and QoL were associated with more frequent CGM use. ${ }^{26}$ On the basis of these findings, we could speculate that patients who use CGM less frequently may feel annoyed with multiple CGM sensor insertions and the alarms. In contrast, compliant patients may perceive more benefits than disadvantages from SAP therapy because it has become part of their daily routine.

It is important for families, when beginning the RT-CGM use, to understand that this technology will not reduce the need to invest effort in diabetes management. SAP therapy, in fact, may be even more time consuming because it forces patients to constantly focus on diabetes care.

Consequently, it is important to offer achievable plans with realistic expectations so that patients can experience some successes from the start of technology use.

Offering first a period of retrospective CGM can enhance SAP therapy acceptance and compliance from patients and families. It could allow the patients to begin CGM without the difficult interpretation of glucose trends. Patients who will find it helpful can then be offered RT-CGM together with CSII for everyday use.

The use of standard reference materials is recommended to teach families to advance from insulin pump to SAP therapy. Also, instructing families in the use of structured algorithms to adjust the insulin regimen can be extremely helpful. Groups with clinical experience in this field have prepared instructions and details on site insertion, taping techniques, and strategies for starting CGM in children and adolescents. ${ }^{28}$ Using these techniques, many very young children can be taught to tolerate CGM well and, in those already on an insulin pump, SAP therapy has been shown to be effective. ${ }^{29}$

\section{Potential barriers to SAP therapy}

Consensus guidelines indicate several potential barriers to SAP therapy adherence. These barriers include: uncertainties about the device accuracy, inadequate reimbursement, the need for educational infrastructure and health care provider support. Other problems that may contribute to SAP therapy abandonment include technical complexities like the role of RT-CGM calibration or how calibration affects accuracy. Patients may also experience frustration when confronted with tasks related to the programming of alerts and management of recorded data. ${ }^{30}$

A good educational program on both the device functions and diabetes management could prevent many of these barriers. Understanding glycemic response to meals, basal/ bolus insulin action, and the need to continue glucometer testing is a necessary requirement for good patient outcome.

We will now describe the most frequent barriers to SAP therapy (Table 2), each one followed by reasonable

Table 2 Common barriers to SAP therapy and potential solutions

\begin{tabular}{|c|c|}
\hline Barriers & Solutions \\
\hline $\begin{array}{l}\text { - Wrong expectations about SAP therapy } \\
\text { (usually believed to be an "artificial pancreas" } \\
\text { by the patient). }\end{array}$ & $\begin{array}{l}\text { Provide the proper training and education before starting SAP therapy (eg, continued } \\
\text { requirement of finger stick testing, possible discordances between RT-CGM and meter } \\
\text { readings, etc). }\end{array}$ \\
\hline - Discomfort from the sensor needle insertion. & $\begin{array}{l}\text { - Use appropriate insertion techniques: insert the sensor in a "pinchable" area and spread } \\
\text { the skin before insertion. Lidocaine-based cream or a cool pack can serve to numb the } \\
\text { area just prior to sensor placement. }\end{array}$ \\
\hline - Low accuracy of glucose calibrations. & $\begin{array}{l}\text { - Clean hands; calibrate during periods when blood glucose levels are not changing rapidly; } \\
\text { calibrate routinely (fasting, premeal, and pre-bedtime). }\end{array}$ \\
\hline - RT-CGM alerts nuisance. & $\begin{array}{l}\text { - Stepwise introduction of alarms. Initial threshold values of } 70 \text { and } 250 \mathrm{mg} / \mathrm{dL} \text {. In the } \\
\text { beginning, do not use any alerts for an initial period of } \mathrm{I}-2 \text { weeks unless the user } \\
\text { frequently experiences hypoglycemia; in such cases, a low alert may be indicated from the } \\
\text { beginning. }\end{array}$ \\
\hline
\end{tabular}

Abbreviations: RT-CGM, real-time continuous glucose monitoring; SAP, sensor-augmented insulin pump. 
tips we believe could improve patient adherence to this therapy on the basis of current clinical guidelines and expert opinions. $^{21,27-30}$

\section{Wrong expectations from SAP therapy}

First and foremost, expectations regarding the product's capabilities are often at odds with its actual features. Many patients contemplating RT-CGM do not fully understand that continued finger stick testing is required for both calibration and confirmation before acting on RT-CGM data. Some users may believe that the system will automatically deliver appropriate insulin dosage, functioning as a closed-loop system (artificial pancreas). Most commonly, many users are also disappointed by discordances between RT-CGM and meter readings, because they expect the two values to match precisely.

- Tip: these drawbacks can be overcome with explanations, training, and education by the health care team on the proper management, use, and limits of this therapy. In particular, it is necessary to explain to the patient that SAP therapy implies more rather than less attention for appropriate diabetes management. ${ }^{7,18}$

\section{The sensor needle insertion}

The use of RT-CGM, in addition to CSII, requires the insertion of a sensor under the belly skin, using a second appropriate anatomic location at some distance from the insulin catheter site (Figure 1). This procedure is quite simple and almost painless, involving the use of an appropriate glucose sensor inserter. After proper training, it can be easily done at home by the patients themselves. However, for several people, this insertion could cause additional pain and discomfort, possibly resulting in glucose sensor use discontinuation.

- Tip: insertion techniques are very important to reduce pain. Sensors should be placed in a "pinchable" area where subcutaneous body fat is present. For those with more abundant subcutaneous fat, it is better to spread the skin until a taut surface for insertion is obtained. Sometimes, lidocaine-based cream can be used on the insertion site 30-45 minutes prior to sensor insertion. Moreover, a cool pack can numb the area just prior to sensor placement. When bleeding occurs, it is better to blot the blood with a cotton swab before connecting the transmitter. $^{27,28}$

\section{Accuracy of CGM calibrations}

The RT-CGM system requires a warm-up period (usually 2 hours) before providing data and periodic calibration thereafter. Timing of the calibration is a fundamental factor to ensure successful use of SAP therapy. Although these patients, before beginning RT-CGM, usually perform selfmonitoring of blood glucose (SMBG) many times every day, many of them are not aware of all of the factors that impact the accuracy of SMBG results. Perhaps a major unrecognized problem is unclean hands. In all RT-CGM systems, calibration requires a sample of blood from clean and dry fingertips. When the capillary glucose measurement for CGM calibration is inaccurate, the device will probably be inaccurate until the next calibration.

Even if current generation RT-CGM must be used in conjunction with SMBG measurements, we frequently see a progressive decrease in SMBG usage once SAP therapy is adopted. Reduced SMBG monitoring may be especially risky near the end of the sensor life, when the accuracy of the RT-CGM may be reduced. It is admittedly difficult to convince some patients to increase their SMBG frequency to pre-CGM levels, but proper calibration is the most important action that patients can take to ensure the accuracy of the sensor data.

As previously reported, there is a discrepancy between the blood glucose level and the interstitial glucose levels measured by the sensor. This discrepancy is greatest when rapid glucose changes occur. It is important to regularly reinforce the patient knowledge that the lag time between blood and interstitial glucose levels can be longer during times of rapid blood glucose changes such as following meals or intense physical activity.

Finally, many patients tend to enter many more blood glucose calibration readings than necessary for the sensor to function properly. Each device has a unique calibration strategy and therefore it is important to follow the manufacturer's instructions for frequency and timing of blood glucose calibrations. ${ }^{28}$

- Tip 1: quality is more important than quantity for calibration. It is better to calibrate less frequently with accurate blood glucose readings during periods when blood glucose levels are not rapidly changing, rather than enter every blood glucose reading at any time. The patients should use the sensor data to confirm that a particular blood glucose check is appropriate for calibration: never calibrate when an arrow indicates a rapid blood glucose rise or fall and review the glucose trend graph to ensure that the glucose levels have remained relatively stable with no wide fluctuations over the 3 hours.

- Tip 2: calibrate routinely rather than waiting for the device to require a calibration. To begin with the RT-CGM use, 
an appropriate calibration schedule includes checking blood glucose fasting in the morning, premeal, and prebedtime (or according to manufacturer's indications).

\section{Managing CGM alerts}

RT-CGM systems provide a large amount of customization for the user and allows for different threshold or predictive alert types. They are designed to alert users when glucose concentrations exceed preset thresholds or when the glucose trend does not meet specified criteria. Threshold alerts are activated when the sensor glucose values exceed or fall below a preset threshold and are easily understood. By contrast, predictive alerts, based on time series data, are usually less clear for the patient and may require additional training before understanding their full value. Predictive alerts are activated when the glucose values recorded by the sensor are predicted to reach a threshold in a preprogrammed time period based on the sensor's rate of change.

Many patients at the beginning tend to set both high and low alert thresholds at levels that result in frequent alerts that will usually result in patient nuisance and possibly in device abandonment.

- Tip: alert settings are very flexible in order to address the user's needs. Each alert can be activated at different times of the day, or can have different thresholds at different times of the day. In addition, each alert has a "repeat" setting that determines how frequently the alert will sound for the same event. Some devices allow a choice of various sounds (or silent vibration) for each different type of alert.

It is important to set the alert thresholds for both high and low levels at appropriate values: this will determine the balance between either a perceived benefit by the patient and therefore long-term use of RT-CGM system or the feeling of annoyance and consequently the discontinuation of sensor use.
In patients already treated with CSII, a phased approach to SAP therapy is crucial in order to obtain maximum adherence to sensor use. As a general rule, Hirsch et al ${ }^{19}$ suggest initial threshold values of 70 and $250 \mathrm{mg} / \mathrm{dL}$ and a stepwise introduction of alarms. Selection of initial alert settings is difficult, and physicians should consider not using alerts for an initial period of 1-2 weeks, unless the patient experiences frequent hypoglycemic episodes; in such cases, a low glucose alert may be indicated from the beginning. This phased approach allows the user to become comfortable with the other aspects of the system maintenance, avoiding frequent high and low glucose alerts. ${ }^{23,30}$

\section{Software reports and telemedicine support}

It is important to mention another RT-CGM-related factor that could increase patient preference and adherence to SAP-therapy. All the available devices are linked to specific software, which allows the transfer of a large amount of data (insulin dosage, carbohydrate intake, eventual exercises, diseases, or other therapies) from the system to a computer (Figure 3 ). This software can easily be used by clinicians and patients to view and evaluate trends, track patterns, and identify areas where therapy adjustments may be necessary, making it easier to compare any changes in glucose levels with the daily activities. By becoming familiar with these software reports, health care providers together with diabetic patients could manage to improve the quality of diabetes management and the patient-related outcomes (making SAP therapy a valuable tool for therapeutic education).

A step forward is the possibility to send the so obtained reports to the health care team electronically, thereby enabling a common and interactive information exchange (telemedicine).

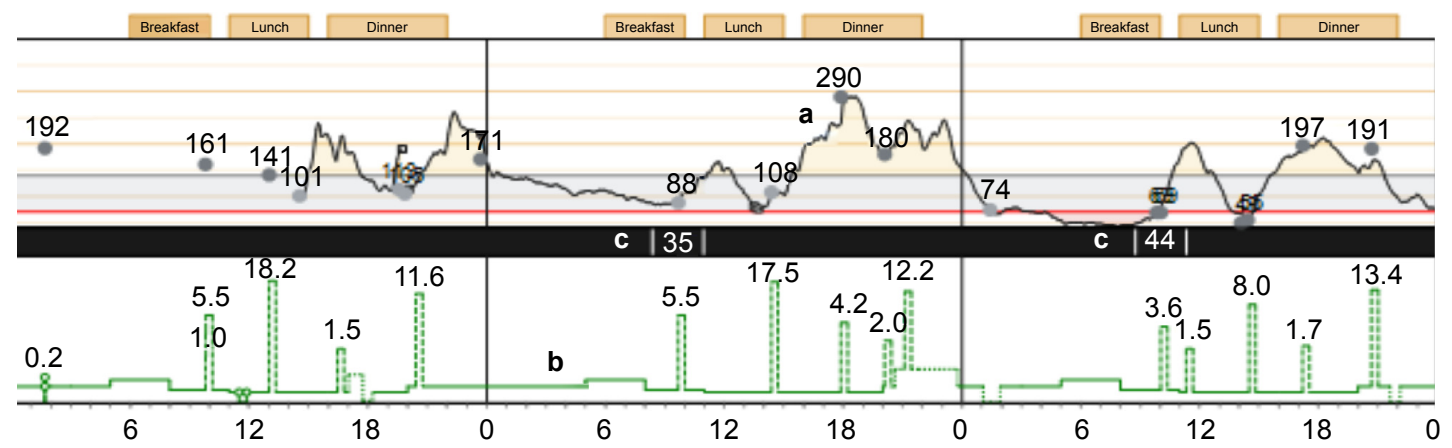

Figure 3 Software reports.

Notes: Software report of a patient on sensor-augmented insulin pump therapy. All the available devices are linked to specific software, which provides the following information about the patient: (a) glucose trend graph, (b) basal and bolus insulin, (c) carbohydrate intake. 


\section{Low-glucose suspend feature and predictive low glucose management (PLGM) for improving adherence}

Health care providers are well aware that severe nocturnal hypoglycemia can be catastrophic and that hypoglycemia remains the most important barrier to improving glycemic control in diabetic patients. Therefore, it could be really helpful to improve the use of threshold-based interruption of insulin pumps in order to ameliorate SAP therapy outcome and adherence. The automatic suspension of insulin delivery when a preset low glucose threshold is reached has been developed since 2009 to mitigate hypoglycemia effect on T1D patients' lives. The low-glucose suspend feature allows for insulin delivery suspension for up to 2 hours at a sensor glucose value that does not require confirmation by the person using the pump. It has been demonstrated that SAP therapy with the threshold-suspend feature is able to reduce nocturnal hypoglycemia without increasing $\mathrm{HbA}_{\mathrm{lc}}$ values. ${ }^{31}$

A step forward in this direction was made in January 2015, when Medtronic announced the launch of the MiniMed 640G System. It represents the next breakthrough toward an artificial pancreas. In fact, advanced in comparison to previous technology, this device uses an algorithm to predict hypoglycemia before it occurs (predictive low glucose management [PLGM]). If hypoglycemia is predicted, the pump will suspend insulin delivery to prevent it from happening. The system then automatically resumes insulin delivery once glucose levels start to recover (thus avoiding hyperglycemic rebound). In the PILGRIM study, testing the PLGM algorithm, impending hypoglycemia was prevented $80 \%$ of the time, with the average suspension of insulin occurring at $92 \mathrm{mg} / \mathrm{dL} .{ }^{32}$

These novel features can play a key role in improving adherence to SAP therapy, especially in those patients experiencing frequent hypoglycemic episodes or hypoglycemia unawareness.

\section{Conclusion}

SAP therapy represents the most advanced technology available today to ameliorate current diabetes care strategies. While current generation RT-CGM systems do not replace current blood glucose meters, they still provide valuable help for the patients and their families. As with most technological advances, the patients need to be aware of both the strengths and limitations of RT-CGM systems. Careful training and troubleshooting strategies will ensure more positive experience and consequent good compliance for patients on SAP therapy. To benefit from glucose trending, more experience and attention is required than the traditional SMBG.
Success with SAP therapy, therefore, requires detailed education and training in diabetes management coupled with extensive training for technicalities in the use of RT-CGM.

Understanding the timing and importance of calibration, how to interpret RT-CGM data during glucose excursions, and how the readings on the CGM receiver can supplement finger stick testing to provide the trend of glucose excursions are all critical issues. Patient acceptance and outcomes will be significantly influenced by judicious choices regarding sensitivity and specificity of alerts. Alert settings must balance the appropriate detection of harmful glycemic events with the number of nuisance alerts issued by the system.

Finally, further prospective studies with large cohorts of patients are needed to systematically assess the main reasons for good or poor adherence to SAP therapy in patients with diabetes. Improvements in our ability to educate patients in the correct understanding of RT-CGM technology and principles, combined with improvements in the product design and reliability, will continue to improve diabetic patients' adherence to SAP therapy in the near future. Educational efforts and good training are the most important ingredients that, combined with the device technology advancement, will lighten the burden of diabetes for patients and their families.

\section{Disclosure}

The authors report no conflicts of interest in this work.

\section{References}

1. Deiss D, Bolinder J, Riveline JP, et al. Improved glycemic control in poorly controlled patients with type 1 diabetes using real-time continuous glucose monitoring. Diabetes Care. 2006;29(12):2730-2732.

2. Juvenile Diabetes Research Foundation Continuous Glucose Monitoring Study Group; Tamborlane WV, Beck RW, et al. Continuous glucose monitoring and intensive treatment of type 1 diabetes. N Engl J Med. 2008; 359(14):1464-1476.

3. Juvenile Diabetes Research Foundation Continuous Glucose Monitoring Study Group; Beck RW, Hirsch IB, et al. The effect of continuous glucose monitoring in well-controlled type 1 diabetes. Diabetes Care. 2009; 32(8):1378-1383.

4. O’Connell MA, Donath S, O'Neal DN, et al. Glycaemic impact of patientled use of sensor-guided pump therapy in type 1 diabetes: a randomised controlled trial. Diabetologia. 2009;52(7):1250-1257.

5. Raccah D, Sulmont V, Reznik Y, et al. Incremental value of continuous glucose monitoring when starting pump therapy in patients with poorly controlled type 1 diabetes: the RealTrend Study. Diabetes Care. 2009; 32(12):2245-2250.

6. Battelino T, Conget I, Olsen B, et al. The use and efficacy of continuous glucose monitoring in type 1 diabetes treated with insulin pump therapy: a randomised controlled trial. Diabetologia. 2012;55(12):3155-3162.

7. Bergenstal RM, Tamborlane WV, Ahmann A, et al. Effectiveness of sensor-augmented insulin pump therapy in type 1 diabetes. $N$ Engl J Med. 2010;363(4):311-320.

8. Hermanides J, Nørgaard K, Bruttomesso D, et al. Sensor-augmented pump therapy lowers HbA1c in suboptimally controlled type 1 diabetes; a randomized controlled trial. Diabet Med. 2011;28(10):1158-1167. 
9. Pickup JC, Freeman SC, Sutton AJ. Glycaemic control in type 1 diabetes during real time continuous glucose monitoring compared with selfmonitoring of blood glucose: meta-analysis of randomised controlled trials using individual patient data. BMJ. 2011;343:d3805.

10. Klonoff DC, Buckingham B, Christiansen JS, et al. Continuous glucose monitoring: an Endocrine Society clinical practice guideline. J Clin Endocrinol Metab. 2011;96(10):2968-2979.

11. Blevins TC, Bode BW, Garg SK, et al. Statement by the American Association of Clinical Endocrinologists Consensus Panel on Continuous Glucose Monitoring. Endocr Pract. 2010;16(5):730-745.

12. Phillip M, Danne T, Shalitin S, et al. Use of continuous glucose monitoring in children and adolescents. Pediatr Diabetes. 2012;13(3): 215-228.

13. Cemeroglu AP, Stone R, Kleis L, etal. Use of a real-time continuous glucose monitoring system in children and young adults on insulin pump therapy: patients' and caregivers' perception of benefit. Pediatr Diabetes. 2010; 11(3):182-187.

14. Barnard KD, Lloyd CE, Skinner TC. Systematic literature review: quality of life associated with insulin pump use in Type 1 diabetes. Diabet Med. 2007;24(6):607-617.

15. Nørgaard K, Scaramuzza A, Bratina N, et al. Routine Sensor-Augmented Pump Therapy in Type 1 Diabetes: The INTERPRET Study. Diabetes Technol Ther. 2013;15(4):273-280.

16. Peyrot M; International DAWN Youth Survey Group. The DAWN Youth WebTalk Study: methods, findings, and implications. Pediatr Diabetes. 2009;10 Suppl 13:37-45.

17. Hirsch IB. Blood glucose monitoring technology: translating data into practice. Endocr Pract. 2004;10(1):67-76.

18. Tumminia A, Crimi S, Sciacca L, et al. Efficacy of real-time continuous glucose monitoring on glycaemic control and glucose variability in type 1 diabetic patients treated with either insulin pumps or multiple insulin injection therapy: a randomized controlled crossover trial. Diabetes Metab Res Rev. 2015;31(1):61-68.

19. Hirsch IB, Abelseth J, Bode BW, et al. Sensor-augmented insulin pump therapy: results of the first randomized treat-to-target study. Diabetes Technol Ther. 2008;10(5):377-383.

20. Tamborlane WV, Beck RV, Laffel L. Continuous glucose monitoring and type 1 diabetes. $N$ Engl J Med. 2009;360(2):190 -192.

21. Hirsch IB. Realistic expectations and practical use of continuous glucose monitoring for the endocrinologist. J Clin Endocrinol Metab. 2009;94(7): $2232-2238$
22. Wentholt IM, Hart AA, Hoekstra JB, Devries JH. Relationship between interstitial and blood glucose in type 1 diabetes patients: delay and the push-pull phenomenon revisited. Diabetes Technol Ther. 2007;9(2): 169-175.

23. Rebrin K, Steil GM. Can interstitial glucose assessment replace blood glucose measurements? Diabetes Technol Ther. 2000;2(3):461-472.

24. Juvenile Diabetes Research Foundation Continuous Glucose Monitoring Study Group. Effectiveness of continuous glucose monitoring in a clinical care environment: Evidence from the Juvenile Diabetes Research Foundation continuous glucose monitoring (JDRF-CGM) trial. Diabetes Care. 2010;33(1):17-22.

25. Mauras N, Beck R, Xing D, et al. A randomized clinical trial to assess the efficacy and safety of real-time continuous glucose monitoring in the management of type 1 diabetes in young children aged 4 to $<10$ years. Diabetes Care. 2012;35(2):204-210.

26. The Diabetes Research in Children Network (DirecNet) Study Group. Psychological aspects of continuous glucose monitoring in pediatric type 1 diabetes. Pediatr Diabetes. 2006;7(1):32-38.

27. Tansey M, Laffel L, Cheng J, et al. Satisfaction with continuous glucose monitoring in adults and youths with Type 1 diabetes. Diabet Med. 2011; 28(9):1118-1122.

28. Ives B, Sikes K, Urban A, Stephenson K, Tamborlane WV. Practical aspects of real-time continuous glucose monitors: the experience of the Yale Children's Diabetes Program. Diabetes Educ. 2010;36(1): 53-62.

29. Frontino G, Bonfanti R, Scaramuzza A, et al. Sensor-augmented pump therapy in very young children with type 1 diabetes: an efficacy and feasibility observational study. Diabetes Technol Ther. 2012;14(9): $762-764$.

30. Hirsch IB, Armstrong D, Bergenstal RM, etal. Clinical application of emerging sensor technologies in diabetes management: consensus guidelines for continuous glucose monitoring (CGM). Diabetes Technol Ther. 2008; 10(4):232-244.

31. Bergenstal RM, Klonoff DC, Garg SK, et al. Threshold-Based InsulinPump Interruption for Reduction of Hypoglycemia. N Engl J Med. 2013; 369(3):224-232.

32. Danne T, TsioliC, KordonouriO, etal. The PILGRIMstudy: in silicomodeling of a predictive low glucose management system and feasibility in youth with type 1 diabetes during exercise. Diabetes Technol Ther. 2014;16(6): $338-347$.
Patient Preference and Adherence

\section{Publish your work in this journal}

Patient Preference and Adherence is an international, peer-reviewed, open access journal that focuses on the growing importance of patient preference and adherence throughout the therapeutic continuum. Patient satisfaction, acceptability, quality of life, compliance, persistence and their role in developing new therapeutic modalities and compounds to optimize

\section{Dovepress}

clinical outcomes for existing disease states are major areas of interest for the journal. This journal has been accepted for indexing on PubMed Central. The manuscript management system is completely online and includes a very quick and fair peer-review system, which is all easy to use. Visit http://www. dovepress.com/testimonials.php to read real quotes from published authors. 